\title{
Sulcal Pattern Variability and Dorsal Anterior Cingulate Cortex Functional Connectivity Across Adult Age
}

\author{
Davide Fedeli, ${ }^{1}$ Nicola Del Maschio, ${ }^{1}$ Camilla Caprioglio, ${ }^{2}$ Simone Sulpizio, ${ }^{1,3}$ and Jubin Abutalebi ${ }^{1,4,5}$
}

\begin{abstract}
Background: The dorsal anterior cingulate cortex (dACC) is a key network hub for cognitive control and environmental adaptation. Previous studies have shown that task-based functional activity in this area is constrained by individual differences in sulcal pattern, a morphologic feature of cortex anatomy determined during fetal life and stable throughout development.

Methods: By using anatomical magnetic resonance imaging and seed-based resting-state functional connectivity (rsFC), we explored the influence of sulcal pattern variability on the functional architecture of the dACC in a sample of healthy adults aged $20-80$ years $(n=173)$.

Results: Overall, rsFC was associated with individual differences in sulcal pattern. Furthermore, rsFC was modulated by the age-sulcal pattern interaction.

Conclusion: Our results suggest a relationship between brain structure and function that partly traces back to early stages of brain development. The modulation of rsFC by the age-sulcal pattern interaction indicates that the effects of sulcal pattern variability on the functional architecture of the dACC may change over adulthood, with potential repercussions for brain network efficiency and cognitive function in aging.
\end{abstract}

Keywords: anterior cingulate cortex; cortical morphology; paracingulate sulcus; resting-state functional connectivity; structure-function relationship; sulcal pattern

\section{Impact Statement}

We investigated for the first time the relationship between anatomical variability of the dorsal anterior cingulate cortex (dACC) and resting-state functional connectivity (rsFC) in young and older adult individuals. Seed-to-voxel rsFC analyses revealed that distinct dACC sulcal patterns are associated with specific profiles of connectivity strength and trajectories, and that this relationship changes as age increases. These findings provide novel insights into the relationship between earlydetermined neuroanatomical variants and brain functional architecture over adulthood.

\section{Introduction}

T He dorsal anterior cingulate cortex (dACC) includes portions of the anterior and middle cingulate cortices on the brain's medial wall (Vogt and PalomeroGallagher, 2012) and is known to mediate a set of regulatory functions critical for cognitive control and environmental adaptation (Botvinick et al., 2001; Bush et al., 2002; Sheth et al., 2012). The functional heterogeneity of the dACC is paralleled by an extensive variability in sulcal pattern, the set of fissures and folds that gives the cortical ribbon its char- acteristic convoluted appearance. The deepest, primary sulci emerge early during fetal life (14th-26th week of gestation) and show remarkable similarity between individuals, suggesting a deterministic imprinting (Borrell, 2018). Secondary and tertiary sulci develop as side-branches of primary sulci between the 30th and 36th week of gestation, yielding variable patterns that are likely influenced by in-womb and perinatal environmental factors (Amiez et al., 2018; Lohmann et al., 1999). Different from quantitative measures of cortex structure (e.g., cortical thickness, surface area, gyrification index), the dACC sulcal pattern is a morphologic feature

\footnotetext{
${ }^{1}$ Centre for Neurolinguistics and Psycholinguistics (CNPL), Università Vita-Salute San Raffaele, Milano, Italy.

${ }^{2}$ Laboratory of Neuroimaging of Aging (LANVIE), University of Geneva, Geneva, Switzerland.

${ }^{3}$ Department of Psychology, University of Milano-Bicocca, Milano, Italy.

${ }^{4}$ The Arctic University of Norway, Troms $\varnothing$, Norway.

${ }^{5}$ Centre for Cognition and Decision Making, National Research University Higher School of Economics, Moscow, Russia.
} 
determined prenatally and stable throughout development (Cachia et al., 2016; Tissier et al., 2018). The longitudinal stability of the dACC sulcal pattern and its resilience to neuroplastic changes* provide a clue to the study of individual differences in structure-function mappings, with the possibility to identify early neuroanatomical constraints on subsequent cognitive abilities (Borst et al., 2014; Fornito et al., 2004; Tissier et al., 2018). Although variability in dACC sulcation has been shown to affect the spatial distribution of local task-related functional activity (Amiez et al., 2013; Crosson et al., 1999; Jahn et al., 2016), it is largely unknown whether differences in sulcal pattern might alter the functional organization of the $\mathrm{AACC}$ in resting state, that is, in absence of overt task performance or stimulation. In the present study, we use structural magnetic resonance imaging (MRI) and seed-based resting-state functional connectivity (rsFC) of healthy adults $(n=173$; age range $=20-80)$ to explore the influence of sulcal pattern variability on the functional architecture of the dACC. Moreover, since the brain's functional connectome encounters substantial changes with increasing age (Chen et al., 2016; Damoiseaux, 2017), we investigate the interaction between dACC sulcal pattern and age on rsFC to inspect for age-related changes in the relationship between local cortical morphology and dACC networks. Finally, we examine whether potential differences in connectivity profiles associated with sulcal pattern variability affect participants' performance on several cognitive test batteries.

Based on previous accounts (Jahn et al., 2016; LopezPersem et al., 2019), we hypothesize that, irrespective of age, seed-based rsFC will be associated with a distinct dACC sulcal pattern. In addition, although current evidence is insufficient to substantiate this prediction, the functional consequences of sulcal pattern variability may vary as a function of age, due to specific interactions between local cortical morphology and $\mathrm{dACC}$ networks in young and older participants. The physiologic mechanisms underlying the reported association between sulcal pattern variability and cognitive control performance are poorly understood (Cachia et al., 2014; Fornito et al., 2004; Huster et al., 2007). Here, we test the hypothesis that the association between dACC sulcal pattern and executive cognitive functions is mediated by the functional architecture of the dACC.

\section{Materials and Methods}

\section{Participants}

Participants' data were drawn from the publicly available Max Planck Institute "Leipzig Study for Mind-BodyEmotion Interactions" (LEMON) data set, which includes neuroimaging and behavioral data from 227 subjects (mean age 38.95; 82 F) (http://fcon_1000.projects.nitrc.org/indi/ retro/MPI_LEMON.html). The study was carried out in accordance with the Declaration of Helsinki; data were collected and analyzed under the local ethics committee approval. Forty-two subjects were excluded because of

\footnotetext{
*Quantitative measures of sulcal anatomy such as sulcal width, depth, or length do change with age and experience (Kochunov et al., 2005; $\mathrm{Li}$ et al., 2010, 2011). However, the interhemispheric distribution of fissures and folds (i.e., the sulcal pattern) has been proven to be longitudinally stable and impervious to neuroplastic processes.
}

psychiatric disorders and/or positivity at drug or alcohol test at time of testing, resulting in a sample of 185 participants (see Table 1 for participants' distribution across age ranges). Participants underwent a detailed imaging protocol consisting of the following: (i) structural MRI scan, that is, a quantitative and weighted T1 magnetization-prepared 2 rapid acquisition gradient echo (MP2RAGE) image, and T2-weighted image fluid-attenuated inversion recovery; (ii) resting-state functional MRI (rs-fMRI); (iii) diffusionweighted imaging; (iv) susceptibility-weighted imaging, and (v) gradient echo field map scans.

Participants also underwent an extensive set of test batteries for cognitive assessment. To investigate whether the relationship between dACC sulcation and cognitive performance is modulated by functional connectivity, we selected tests tapping verbal and nonverbal cognitive processes that engage the dACC: California Verbal Learning Task (CVLT), Test of Attentional Performance (TAP), Trail Making Test (TMT), Wortschatztest (WST), Leistungsprüfsystem 2 (LPS 2), and Regensburger Word Fluency Test (RWT). For a detailed description of the test batteries, see Supplementary Data.

\section{$M R I$ acquisition}

MP2RAGE sequences were acquired with a 3 Tesla scanner (MAGNETOM Verio; Siemens Healthcare GmbH, Erlangen, Germany) with the following parameters: repetition time $(\mathrm{TR})=5000 \mathrm{~ms}$, echo time $(\mathrm{TE})=2.92 \mathrm{~ms}$; flip angle $1=4^{\circ}$ flip angle $2=5^{\circ}$, field of view $(F O V)=256 \mathrm{~mm}$, number of slices $=176$, voxel size $=1 \mathrm{~mm}^{3}$ isotropic, GRAPPA acceleration factor $=3$, slice order $=$ interleaved. T2 $*$-weighted gradient echo planar imaging multiband BOLD scans were acquired with the following parameters: $\mathrm{TR}=1400 \mathrm{~ms}$, $\mathrm{TE}=$ $30 \mathrm{~ms}$, flip angle $=69^{\circ}, \mathrm{FOV}=202 \mathrm{~mm}$, number of slices $=64$, voxel size $=2.3 \mathrm{~mm}^{3}$ isotropic, imaging matrix $=88 \times 88$, slice thickness $=2.3 \mathrm{~mm}$, echo spacing $=0.67 \mathrm{~ms}$, bandwidth $=$ $1776 \mathrm{~Hz} /$ pixel, partial Fourier 7/8, multiband acceleration factor $=4$, volumes $=657$, slice order $=$ interleaved. For further details on MRI acquisition parameters, see Babayan and colleagues (2018).

\section{dACC sulcal pattern classification}

Differences in dACC sulcation are primarily assessed by quantifying the occurrence and characteristics of the paracingulate sulcus (PCS), a variable secondary sulcus that runs

Table 1. Participants' Frequencies for Each Age RANGE SPLIT BY GENDER

\begin{tabular}{lrrr}
\hline Age range, years & \multicolumn{1}{c}{$\boldsymbol{M}$} & $M$ & Total \\
\hline $20-25$ & 23 & 43 & 66 \\
$25-30$ & 8 & 37 & 45 \\
$30-35$ & 3 & 7 & 10 \\
$55-60$ & 2 & 2 & 4 \\
$60-65$ & 6 & 11 & 17 \\
$65-70$ & 14 & 9 & 23 \\
$70-75$ & 8 & 9 & 17 \\
$75-80$ & 2 & 1 & 3 \\
Total & 66 & 119 & 185 \\
\hline
\end{tabular}

F, female; M, male. 
dorsal and parallel to the cingulate sulcus (CS) on the medial surface of the ACC (Ono et al., 1990; Paus et al., 1996; Yücel et al., 2001). Here, following Ono and colleagues' nomenclature (Ono et al., 1990), as in our previous studies (Cachia et al., 2017; Del Maschio et al., 2019), two types of dACC sulcation were identified depending on the PCS occurrence in each hemisphere: a "single" type (CS only) and a "double parallel" type (CS and additional PCS) (Fig. 1a, b). An asymmetry index based on this classification was used to assess the interhemispheric distribution of the PCS in each brain, yielding four sulcal patterns: (i) "single/single" (SS) (CS only in both hemispheres); (ii) "double parallel/double parallel" (DD) (CS and additional PCS in both hemispheres); (iii) "leftward asymmetry" (LA) (PCS present in the left hemisphere only); and (iv) "rightward asymmetry" (RA) (PCS present in the right hemisphere only).

All structural images were visually inspected for the presence of artifacts (e.g., excessive head movement, ghosting, field inhomogeneities or distortions) and six participants were excluded due to this reason. Moreover, two participants were excluded due to the presence of a calcified meningioma as assessed by an expert neurologist. Overall, T1-weighted MR images were obtained from 177 participants. The origin was set on all individual structural images to match the anterior commissure-posterior commissure (bicommissural) line; subsequently, segmentation and skull-stripping were performed by means of Statistical Parametric Mapping (SPM) 12 (version 6906).

Following Garrison's PCS measurement protocol (Garrison, 2017), first reported in Garrison and colleagues (2015), all T1 images were imported into MANGO (Multiimage Analysis GUI, v 4.0, http://ric.uthscsa.edu/mango/ mango.html, The University of Texas Health Science Center; see also Del Maschio et al., 2019). The CS was identified at $4 \mathrm{~mm}$ laterally from the midline $(\mathrm{x}=0)$ on either hemisphere, lying dorsal to the corpus callosum. The PCS was identified as the sulcus running dorsal and parallel to the CS. The anterior limit of the PCS was identified at $x= \pm 5 \mathrm{~mm}$, corresponding to the point at which the sulcus begins to move posteriorly from the anterior commissure and runs parallel to the CS for most of its length until it encounters an imaginary line perpendicular to the bicommissural line and passing through the anterior commissure. In each hemisphere, the PCS was classified as "present" ( $\geq 20 \mathrm{~mm})$ or "absent" $(\leq 19 \mathrm{~mm})$. In case of PCS discontinuity, sulcus fragments were included in the analysis only if interruptions were $\leq 19 \mathrm{~mm}$. At this stage, spatial normalization was not performed.

PCS measurement and dACC sulcal pattern classification, incorporating both visual inspection and automated components, were carried by three expert blind raters (inter-rater
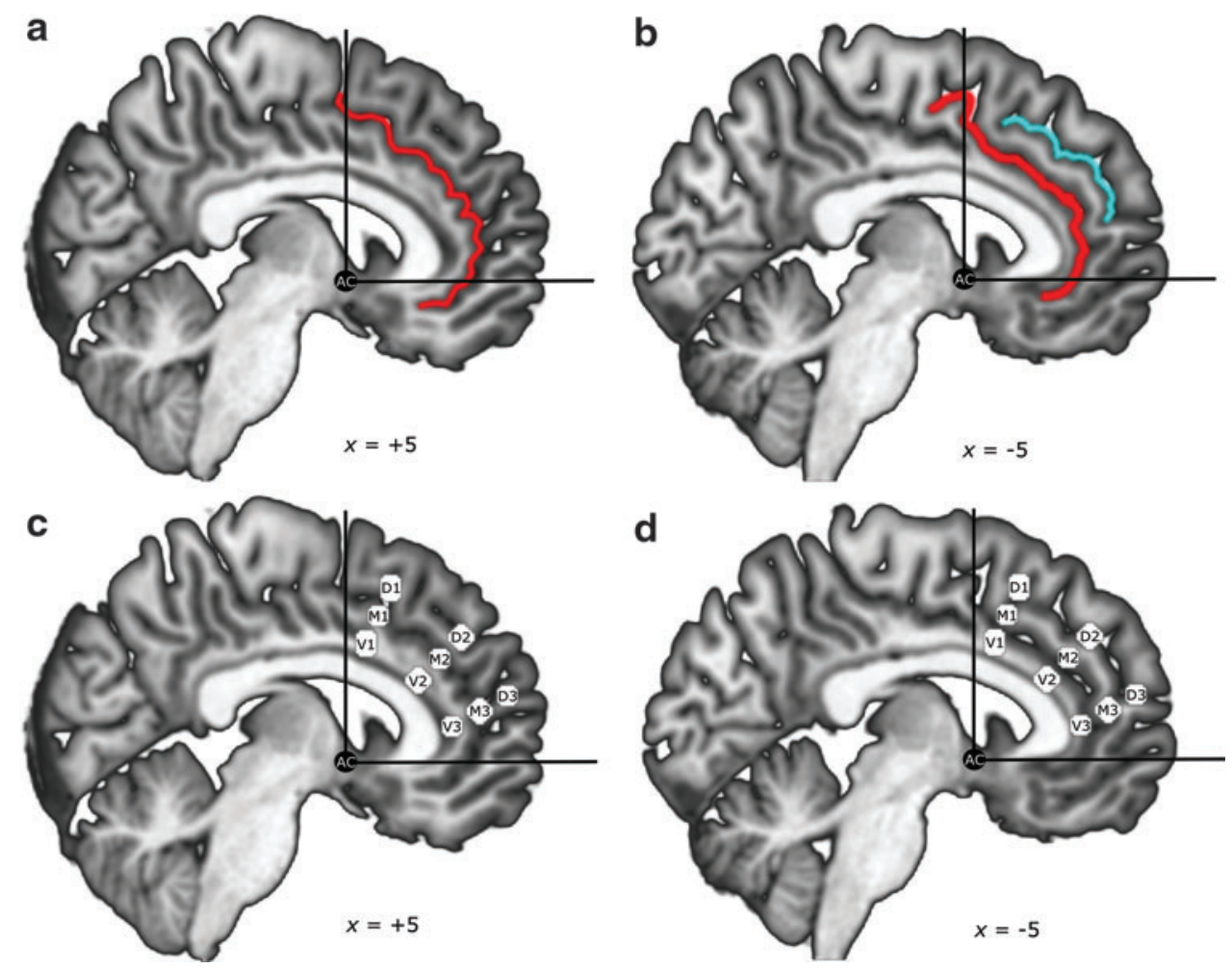

FIG. 1. The two hemispheres of the skull-stripped brain of the same individual (sub-032304) are shown. (a) ACC with a single pattern (CS only) in MNI space. The anterior branch of the CS is marked in red. (b) ACC with a double parallel pattern (CS and additional PCS) in MNI space. The anterior branch of the CS is marked in red, and PCS is marked in light blue. (c, d) Show the seeds used for seed-to-voxel resting-state functional connectivity analyses. Ventral seeds MNI $X, Y, Z$ coordinates: $\mathrm{V} 1( \pm 5,10,33)$; V2 $( \pm 5,27,21) ; \mathrm{V} 3( \pm 5,38,6)$. Middle seeds MNI $X, Y, Z$ coordinates: M1 $( \pm 5,14,42)$; M2 ( $\pm 5,34,28) ;$ M3 $( \pm 5,47,11)$. Dorsal seeds MNI $X, Y, Z$ coordinates: D1 $( \pm 5,18,51)$; D2 $( \pm 5,41,35)$; D3 ( $\pm 5,56,16)$. ACC, anterior cingulate cortex; CS, cingulate sulcus; PCS, paracingulate sulcus. Color images are available online. 
agreement $=97 \%$ ). Disagreement among raters due to ambiguous dACC sulcation (Leonard et al., 2009) was resolved by a fourth blind rater. Since ambiguity persisted after general inter-rater discussion for 4 participants (3\% of the whole data set), those participants were excluded from subsequent analyses, yielding a final sample of 173 participants.

\section{Seed-to-voxel rsFC}

Data preprocessing and rsFC analyses were run with CONN toolbox (version 18.b) (Whitfield-Gabrieli and Nieto-Castanon, 2012) in SPM12. Using the default preprocessing pipeline implemented in CONN, functional images were slice-timing corrected, realigned to the first image, unwarped, and centered to $(0,0,0)$ coordinates. Outlier volumes with excessive head motion were detected and scrubbed using the Artifact Rejection Toolbox (ART). Acquisitions with framewise displacement above $0.9 \mathrm{~mm}$ or global BOLD signal changes above 5 s.d. (over 95th percentile) were flagged as potential outliers. We allowed for scrubbing of up to $20 \%$ of functional volumes per subject. However, no subject in the final sample had to be discarded because of this reason. Structural images were segmented and normalized with the unified segmentation-normalization approach with default parameters (Ashburner and Friston, 2005). Functional images were normalized to average template in MNI space and smoothed with a full-width at half-maximum $8 \mathrm{~mm}$ Gaussian kernel. Since noise removal increases the reliability of rs-fMRI data, the anatomical component-based noise correction method (aCompCor) was applied to extract a representative noise signal from white matter (WM) and cerebrospinal fluid (CSF) components on a voxel-by-voxel level (Chai et al., 2012; Behzadi et al., 2007).

For each participant, realignment, scrubbing parameters, noise components from WM and CSF, and "effect of rest" were entered as nuisance covariates in the first-level analysis (Nieto-Castañón, 2020). Realignment parameters are defined as 12 components estimated from the subject motion parameters ( 3 translation, 3 rotation, and their first-order temporal derivatives). Scrubbing parameters consist in subjectspecific dummy variables indicating outlier volumes identified with ART (Power et al., 2012). Physiological noise components from WM and CSF are defined as the significant principal components derived from nongray-matter "noise" regions in which the signal is supposed not to be modulated by the local neural activity (Behzadi et al., 2007). At first, WM and CSF masks are computed subject-wise by creating eroded-by-one-voxel binary masks of all the voxels with values greater than $50 \%$ within WM and CSF tissue probability maps. Afterward, five noise components within each mask are estimated. The first consisting the average BOLD signal and the other four as the result of a principal component analysis (PCA) following the aCompCor method as implemented by Chai and colleagues (2012) (see also Nieto-Castañón, 2020). In addition, a small trend at the very beginning of each participant's functional session (defined as "effect of rest" or "session effect") is automatically estimated by CONN. This regressor corresponds to a step function convolved with the hemodynamic response function that allows to control for potential initial magnetization transient effects (or "ramping effects") at the very beginning of the session, before the BOLD signal acquisition reaches the steady-state behavior. Subsequently to the linear regression of potential confounding effects, functional images were bandpassfiltered $(0.01-0.1 \mathrm{~Hz})$ to remove low-frequency drifts and physiological high-frequency noise.

A seed-to-voxel approach was implemented in CONN toolbox. Following Margulies and colleagues (2007), three parallel lines of spherical seeds (radius $=4 \mathrm{~mm}$ ) were defined along the dorsal-to-caudal gradient of the dACC: (i) the ventral seed line lies $5 \mathrm{~mm}$ above the ACC callosal curve in the ACC; (ii) the middle seed line lies $15 \mathrm{~mm}$ above the callosal curve in the ACC, extending to the inferior part of the paracingulate gyrus (PCG), as defined by the anatomical mask of the probabilistic Harvard-Oxford atlas (distributed with the FMRIB Software Library FSL, Smith et al., 2004); and (iii) the dorsal seed line lies $25 \mathrm{~mm}$ above the callosal curve in the cingulo-frontal transition area, corresponding to the superior part of the PCG of the Harvard-Oxford atlas. ${ }^{\dagger}$ One set of seeds, comprising the three parallel lines of spherical seeds, was created for each hemisphere at $x= \pm 5 \mathrm{~mm}$ (see Fig. 1c, d, where also the MNI coordinates of the seeds are reported). A seed-to-voxel analysis with spherical seeds was preferred to selecting seed regions of interest (ROIs) based on anatomical parcellation. Indeed, anatomical variability (with specific reference to the cingulate cortex sulcation patterns) represents a source of large interpackage differences in brain parcellation schemes implemented in popular neuroimaging software, which may consequently jeopardize result generalizability and compatibility (Mikhael et al., 2018).

A whole-brain bivariate correlation analysis was carried out between the BOLD time-series of each seed (i.e., signals average across all voxels within the seed) and the BOLD time-series of all other voxels of the brain. The Pearson's correlation coefficients obtained were then converted to normally distributed scores using Fisher's $R$ to $Z$ transform to allow for second-level general linear model analysis. Significance threshold was set at voxel- $p$-uncorrected $<0.001$ and cluster- $p$-family-wise error (FWE)-corrected for multiple comparisons $<0.05$.

\section{Statistical analyses}

Statistical analyses were conducted to (i) explore the distribution of the dACC sulcal pattern in our sample; (ii) investigate the effects of dACC sulcal pattern variability on seed-based rsFC; (iii) assess whether the relationship between dACC sulcal pattern and seed-based rsFC changes with increasing age; and (iv) estimate correlations between connectivity profiles associated with distinct sulcal patterns and cognitive performance. Analyses were performed with CONN toolbox and SPSS software (version 21; IBM Corp. Released 2012).

\section{dACC sulcal pattern distribution}

Incidence rates (\%) of dACC sulcal pattern were calculated for all participants and compared with equal frequency distribution by means of chi-square $\left(\chi^{2}\right)$ goodness of fit. Since evidence of gender effects on sulcal pattern distribution is mixed (cf. Del Maschio et al., 2019; Leonard et al.,

\footnotetext{
${ }^{\dagger}$ Anatomically, the dorsal seeds lie in a cingulo-frontal region known as "paralimbic belt" or "paracingulate gyrus" when a PCS is present (Fornito et al., 2004).
} 
2009; Yücel et al., 2001), an ordinal logistic regression model was run to explore the distribution of the dACC sulcal pattern with gender as predictor (reference level: male) and dACC sulcal pattern as dependent variable ("single/single," "double parallel/double parallel," "leftward asymmetry," "rightward asymmetry").

\section{Effects of dACC sulcal pattern variability on seed-based rsFC}

To investigate the effects of individual differences in dACC sulcation on dACC connectivity, a general linear model was run with dACC sulcal pattern as regressors ("single/single," "double parallel/double parallel," "leftward asymmetry," "rightward asymmetry"); age, gender, and education as nuisance covariates; and seed-based rsFC as a dependent variable. For each dACC seed (nine per hemisphere), the following paired differences $t$-contrasts were computed: (i) single/single $>$ double parallel/double parallel; (ii) single/single >leftward asymmetry; (iii) single/single > rightward asymmetry; (iv) double parallel/double parallel> leftward asymmetry; (v) double parallel/double parallel> rightward asymmetry; and (vi) leftward asymmetry>rightward asymmetry. When each contrast was performed, other seeds were not covaried out. Connectivity differences between dACC sulcal patterns were tested each time for all the included seeds. Given the high number of performed univariate tests (i.e., 9 seeds*2 hemispheres*6 comparisons $=108 t$-tests), the cluster-level FWE-corrected $p$-value threshold was additionally divided by the number of performed $t$-tests (i.e., $0.05 / 108=0.00046$ ).

Task-based fMRI studies have indicated topological modulations of clusters of brain activity based on the sulcal morphology of the dACC. When interpreting results from an fMRI decision-making task, Amiez and colleagues (2013) have suggested that the CS (when a PCS is absent) and the PCS (when present) are functionally homologous. To test this hypothesis on $\mathrm{rsFC}$, we have performed an additional analysis by comparing dorsal (D) and middle (M) seeds in individuals with and without a PCS. Unthresholded connectivity maps were generated to inspect spatial overlaps between $\mathrm{M}$ seeds and $\mathrm{D}$ seeds in the double parallel/double parallel and single/single groups. Connectivity differences were investigated by means of pairwise $t$-tests selecting middle seeds for the single/single group (i.e., M1, M2, M3) and dorsal seeds for the double parallel/double parallel group (i.e., D1, D2, D3) (Supplementary Data, Supplementary Table S1 and Supplementary Fig. S1).

\section{Effects of age-sulcal pattern interaction on seed-based rsFC}

To inspect for age-dependent changes in the relationship between dACC sulcal pattern and seed-based rsFC, a general linear model was run with dACC sulcal pattern*age as regressors (single/single*age; double parallel/double parallel* age; leftward asymmetry*age; rightward asymmetry*age), gender and education as nuisance covariates, and seedbased $\mathrm{rsFC}$ as a dependent variable. Contrasts were the same as those computed to investigate the effects of dACC sulcal pattern variability on seed-based rsFC. To further explore the interaction effects, significant connectivity changes that emerged from the age-sulcal pattern in- teraction analyses were investigated by splitting our sample into younger (age <35) and older adults (age >60) (Supplementary Data and Supplementary Table S2).

\section{Correlations between $\mathrm{dACC}$ connectivity and cognitive performance}

To test for cognitive effects of sulcal-related differences in dACC connectivity, correlational analyses were performed between dACC connectivity values associated with distinct sulcal patterns and performance at the following test batteries: CVLT, TAP, TMT, WST, LPS 2, and RWT. For each significant cluster resulting from the seed-to-voxel rsFC analyses, individual connectivity values were extracted. Correlations between cognitive scores and individual connectivity values were performed group-wise (e.g., for a cluster resulting from $\mathrm{SS}>\mathrm{DD}$ contrast, connectivity scores of individuals with SS pattern and DD pattern were separately correlated with each of the selected cognitive tests). In case of significant correlations resulting from the same cluster with the same cognitive test, correlations coefficients were compared between groups. Following guidelines for improving brain-behavior correlations (Rousselet and Pernet, 2012), all analyses were performed with the freely available MATLAB toolbox "Robust Correlation Toolbox" (http://sourceforge.net/projects/robustcorrtool/) (Pernet et al., 2013), which allows assumption checking and outlier detection, and implements robust correlations (e.g., percentage-bend correlation and skipped correlation, see Wilcox, 1994, 2004). To prevent the risk of false-positive errors, all analyses were corrected for multiple comparisons.

\section{Results}

\section{dACC sulcal pattern distribution}

Absolute numbers and proportions of dACC sulcal pattern across participants and split by gender are reported in Table 2. The "leftward asymmetry" pattern occurred more frequently than others in our sample [LA $=32.37 \%$; $\mathrm{SS}=27.75 \% ; \mathrm{DD}=20.81 \% ; \mathrm{RA}=19.7 \% ; \chi^{2}(3)=7.9249$, $p=0.04759$ ] confirming a well-known leftward bias in the general population (Fornito et al., 2004; Huster et al., 2007; Paus et al., 1996; Wei et al., 2017). No significant effect of gender was detected on dACC sulcal pattern distribution $(\beta=-0.280$, st.err. $=0.301, p>0.3)$.

Table 2. Sulcal Pattern Distribution IN THE SAMPLE $(N=173)$

\begin{tabular}{lcrc}
\hline $\begin{array}{l}\text { dACC sulcal } \\
\text { pattern }\end{array}$ & $\begin{array}{c}\text { Overall } \\
(\mathrm{N}=173)\end{array}$ & $F(\mathrm{~N}=60)$ & $M(\mathrm{~N}=113)$ \\
\hline SS & $48(27.75)$ & $23(38.33)$ & $25(22.13)$ \\
DD & $36(20.81)$ & $9(15.00)$ & $27(23.89)$ \\
LA & $56(32.37)$ & $15(25.00)$ & $41(36.28)$ \\
RA & $33(19.07)$ & $13(21.67)$ & $20(17.70)$ \\
\hline
\end{tabular}

Incidence rates $(\%)$ are calculated within groups and reported in brackets.

CS, cingulate sulcus; dACC, dorsal anterior cingulate cortex; DD, double parallel/double parallel' (CS and additional PCS in both hemispheres); F, female; LA, leftward asymmetry (PCS present in the left hemisphere only); M, male; PCS, paracingulate sulcus; RA, rightward PCS asymmetry (PCS present in the right hemisphere only); SS, single/single (CS only in both hemispheres). 
Table 3. Paired Differences t-Contrast Results for the Main Effect of Dorsal Anterior Cingulate Cortex Sulcal Pattern on Seed-Based Functional Connectivity

\begin{tabular}{|c|c|c|c|c|c|c|c|c|c|c|}
\hline \multirow{2}{*}{$\frac{\text { Contrast }}{\mathrm{SS}>\mathrm{DD}}$} & \multirow{3}{*}{$\begin{array}{l}\text { Seed } \\
\text { V2 L } \\
\text { M3 L }\end{array}$} & \multicolumn{3}{|c|}{$\begin{array}{l}\text { MNI coordinates } \\
(\mathrm{X}, \mathrm{Y}, \mathrm{Z})\end{array}$} & \multirow{2}{*}{$\begin{array}{l}\text { Harvard-Oxford } \\
\text { Atlas region }\end{array}$} & \multirow{2}{*}{$\begin{array}{c}\text { Connectivity } \\
\text { increasel } \\
\text { decrease } \\
-\end{array}$} & \multirow{2}{*}{$\frac{k}{372}$} & \multirow{2}{*}{$\frac{Z \text { score }}{5.2}$} & \multirow{2}{*}{$\begin{array}{c}\begin{array}{c}\text { Cluster-level } \\
\text { p-FWE }\end{array} \\
0.000335\end{array}$} & \multirow{3}{*}{$\begin{array}{c}\begin{array}{c}\text { Voxel-level } \\
\text { p-FWE }\end{array} \\
0.017112\end{array}$} \\
\hline & & -30 & 10 & -10 & & & & & & \\
\hline & & -26 & -84 & -40 & Cerebellum & + & 660 & 4.47 & 0.000011 & \\
\hline $\mathrm{SS}>\mathrm{LA}$ & - & - & - & - & - & - & - & - & - & 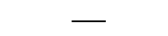 \\
\hline \multirow[t]{4}{*}{$\mathrm{SS}>\mathrm{RA}$} & V1 R & 36 & -74 & 18 & $\begin{array}{l}\text { Lateral occipital cortex, } \\
\text { superior division }\end{array}$ & - & 702 & 4.65 & 0.000003 & 0.163567 \\
\hline & & -36 & -82 & 14 & $\begin{array}{l}\text { Lateral occipital cortex, } \\
\text { superior division }\end{array}$ & - & 621 & 4.76 & 0.000010 & 0.107210 \\
\hline & & 24 & -50 & -16 & $\begin{array}{l}\text { Temporal occipital } \\
\text { fusiform cortex }\end{array}$ & - & 436 & 4.55 & 0.000202 & 0.235664 \\
\hline & & 8 & -64 & -22 & Cerebellum & - & 396 & 4.73 & 0.000410 & 0.118297 \\
\hline \multirow[t]{4}{*}{$\mathrm{DD}>\mathrm{LA}$} & V3 R & 62 & -52 & 28 & Angular gyrus & + & 456 & & 0.000069 & 0.071920 \\
\hline & D3 L & 32 & 6 & 16 & Insular cortex & + & 552 & 4.2 & 0.000025 & 0.576600 \\
\hline & & -56 & -14 & 12 & Central opercular cortex & + & 548 & 4.27 & 0.000026 & 0.556079 \\
\hline & & -36 & -4 & -4 & Insular cortex & + & 405 & 4.48 & 0.000306 & 0.306549 \\
\hline $\mathrm{DD}>\mathrm{RA}$ & - & - & - & - & - & - & - & - & - & - \\
\hline $\mathrm{LA}>\mathrm{RA}$ & - & - & - & - & - & - & - & - & - & - \\
\hline
\end{tabular}

Significance threshold is set at voxel- $p$-uncorrected $<0.001$ and cluster- $p$-FWE-corrected $<0.00046$. Voxel- $p$-FWE $<0.05$ corrected values are reported for additional information. Only one local maximum per significant cluster is reported.

FWE, family-wise error.

\section{Effects of $d A C C$ sulcal pattern variability on seed-based rsFC}

Individual differences in sulcal pattern were associated with distinct profiles of functional organization of the dACC. Results for each sulcal pattern are described below, reported in Table 3, and illustrated in Figure 2.

\section{Single/single (CS only in both hemispheres)}

The single/single $>$ double parallel/double parallel contrast showed reduced rsFC of a ventral seed (V2 L) with the left insular cortex. Enhanced long-distance, intrahemispheric rsFC was found between a left middle (M3 L) seed and the cerebellum. The single/single>leftward asymmetry contrast was not associated with any significant difference. The single/single $>$ rightward asymmetry contrast revealed extensive patterns of reduced long-distance rsFC between a right ventral seed (V1 R) and bilateral occipital cortices, as well as right temporo-occipital and cerebellar regions.

\section{Double parallel/double parallel (CS and PCS in both hemispheres)}

The double parallel/double parallel>single/single contrast showed enhanced rsFC of a ventral seed (V2 L) with the left insula. Reduced long-distance, intrahemispheric rsFC was found between a left middle (M3 L) seed and the cerebellum. The double parallel/double parallel>leftward asymmetry contrast showed enhanced intrahemispheric rsFC between a right ventral seed (V3 R) and the right angular gyrus, as well as between a left dorsal seed (D3 L) and bilateral insulo-opercular cortices. No difference in rsFC was found for the double parallel/double parallel $>$ rightward asymmetry contrast.

\section{Leftward asymmetry (PCS in the left hemisphere only)}

The leftward asymmetry $>$ single/single contrast showed no significant differences for any of the considered seeds. The leftward asymmetry>double parallel/double parallel contrast resulted in reduced intrahemispheric rsFC between a right ventral seed (V3 R) and the right angular gyrus, as well as between a left dorsal seed (D3 L) and bilateral insulo-opercular cortices. The leftward asymmetry>rightward asymmetry contrast showed no significant result.

\section{Rightward asymmetry (PCS in the right hemisphere only)}

The rightward asymmetry $>$ single/single contrast revealed extensive patterns of enhanced long-distance rsFC between a right ventral (V1 R) seed and bilateral occipital cortices, as well as right temporo-occipital and cerebellar regions. No difference in $\mathrm{rsFC}$ was found for the rightward asymmetry $>$ double parallel/double parallel contrast. The rightward asymmetry>leftward asymmetry contrast showed no significant difference as well.

\section{Effects of age-sulcal pattern interaction on seed-based rsFC}

The relationship between dACC sulcal pattern and dACC connectivity changed with increasing age, although to varying degree depending on the specific sulcal pattern considered. Results for each sulcal pattern are described below, reported in Table 4, and represented in Figure 3.

\section{Single/single*age}

The single/single*age $>$ double parallel/double parallel*age contrast showed increased long-distance, intrahemispheric rsFC between a left middle (M3L) seed and the cerebellum. The single/single*age $>$ leftward asymmetry*age contrast showed increased rsFC between a right ventral (V3 R) seed with the temporal cortex. The single/single*age $>$ rightward asymmetry*age contrast showed no significant result. 
a

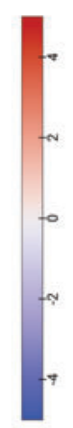

b

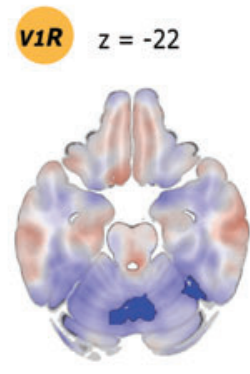

$S S>D D$
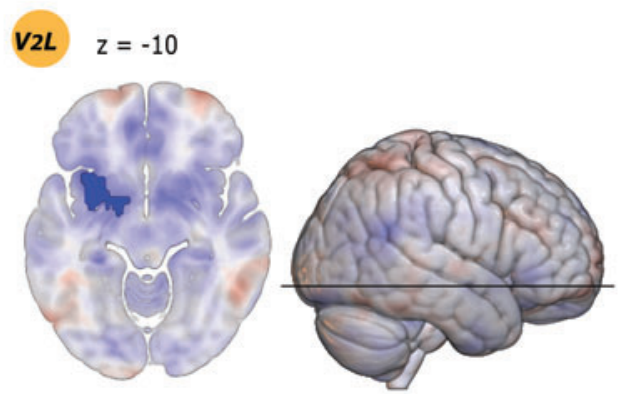
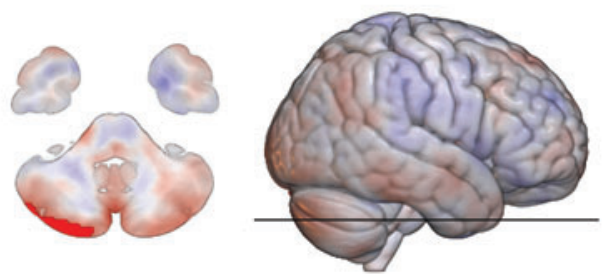

SS > RA
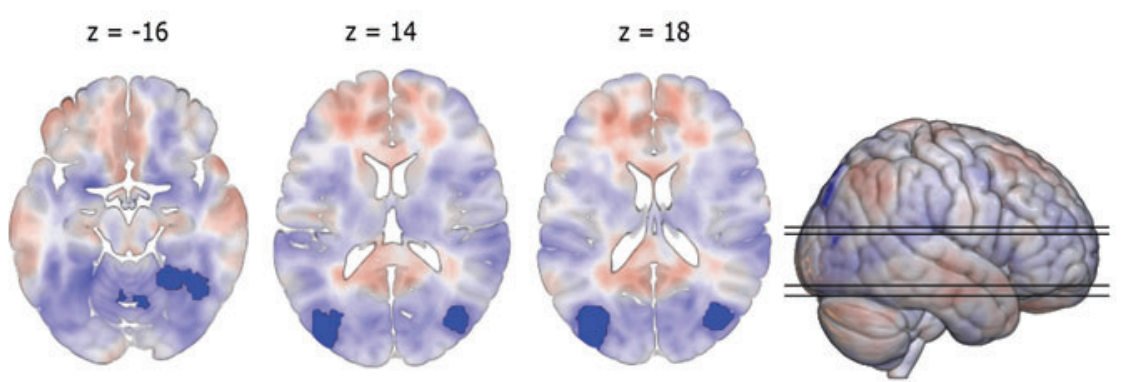

C

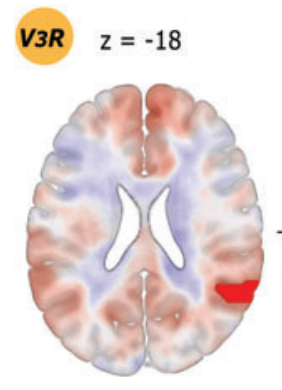

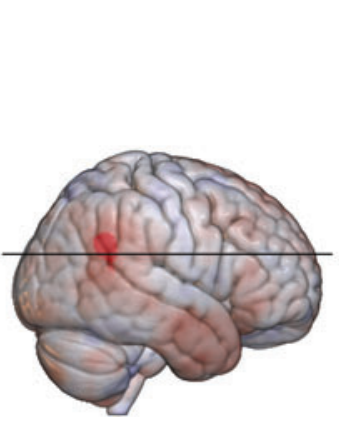

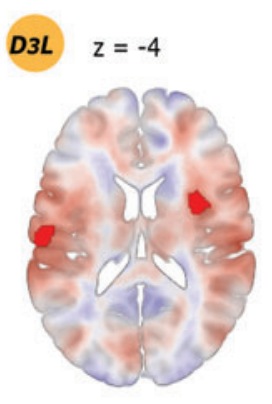

$D D>L A$
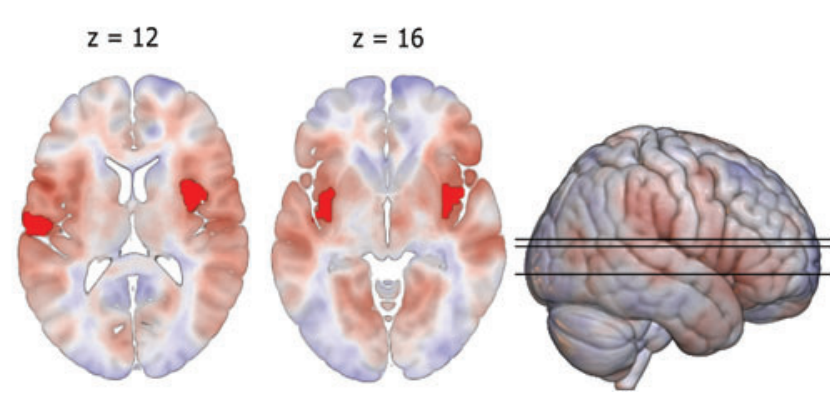

FIG. 2. Effects of dACC sulcal pattern variability on seed-based resting-state functional connectivity. Results are reported as highlighted clusters overlayed onto unthresholded T-maps; color map indicates raw $t$-values (Pernet and Madan, 2020). Significant results are shown at cluster-level FWE-corrected for multiple comparisons $p$-value $<0.00046$, and voxel-leveluncorrected $p$-value $<0.001$. Axial slices are shown with $Z$ MNI coordinates corresponding to the contrast local maxima with whole-brain height reference. Ventral seeds are labeled as V1, V2, V3; middle seeds are labeled as M1, M2, M3; dorsal seeds are labeled as D1, D2, D3. Images are in neurological display convention. dACC, dorsal anterior cingulate cortex; DD, double parallel/double parallel' (CS and additional PCS in both hemispheres); FWE, family-wise error; L, left hemisphere; LA, leftward asymmetry (PCS present in the left hemisphere only); R, right hemisphere; RA, rightward PCS asymmetry (PCS present in the right hemisphere only); SS, single/single (CS only in both hemispheres). Color images are available online.

Table 4. Paired Differences $T$-Contrast Results of the Interaction Between Age and Anterior Cingulate Cortex Sulcal Pattern

\begin{tabular}{|c|c|c|c|c|c|c|c|c|c|c|}
\hline \multirow{3}{*}{$\begin{array}{l}\text { Contrast } \\
\text { SS*age }>\text { DD*age } \\
\text { SS*age }>\text { LA*age }\end{array}$} & \multirow{2}{*}{$\begin{array}{l}\text { Seed } \\
\text { M3 L }\end{array}$} & \multicolumn{3}{|c|}{$\begin{array}{c}\text { MNI coordinates } \\
\quad(\mathrm{X}, \mathrm{Y}, \mathrm{Z})\end{array}$} & $\begin{array}{l}\text { Harvard-Oxford } \\
\text { Atlas region }\end{array}$ & $\begin{array}{c}\text { Connectivity } \\
\text { increasel } \\
\text { decrease }\end{array}$ & $k$ & $Z$ score & $\begin{array}{c}\text { Cluster-level } \\
\text { p-FWE }\end{array}$ & $\begin{array}{l}\text { Voxel-level } \\
\text { p-FWE }\end{array}$ \\
\hline & & -18 & -86 & -42 & Cerebellum & + & 628 & 4.53 & 0.000018 & 0.234223 \\
\hline & V3 R & 56 & -2 & -18 & $\begin{array}{l}\text { Middle temporal gyrus, } \\
\text { anterior division }\end{array}$ & + & 554 & 4.89 & 0.000022 & 0.061480 \\
\hline SS*age $>$ RA*age & - & - & - & - & - & - & - & - & - & - \\
\hline \multirow[t]{3}{*}{ DD*age $>$ LA*age } & M1 L & -36 & -16 & 22 & Central opercular cortex & + & 394 & 5.07 & 0.000190 & 0.030792 \\
\hline & D3 L & -56 & -18 & 14 & Central opercular cortex & + & 513 & 4.38 & 0.000048 & 0.411159 \\
\hline & & 38 & 6 & 16 & Central opercular cortex & + & 429 & 4.22 & 0.000204 & 0.620230 \\
\hline DD*age $>$ RA*age & V1 R & 8 & 50 & -4 & Paracingulate gyrus & + & 587 & 4.39 & 0.000020 & 0.399625 \\
\hline \multirow[t]{2}{*}{ LA*age $>$ RA*age } & D2 R & 6 & 40 & 2 & $\begin{array}{l}\text { Cingulate gyrus, } \\
\text { anterior division }\end{array}$ & - & 1081 & 4.61 & 0.000000 & 0.192153 \\
\hline & & 0 & -56 & 50 & Precuneus cortex & - & 404 & 3.84 & 0.000334 & 0.971300 \\
\hline
\end{tabular}

Significance threshold is set at voxel- $p$-uncorrected $<0.001$ and cluster- $p$-FWE-corrected $<0.00046$. Voxel- $p$-FWE $<0.05$ corrected values are reported for additional information. Only one local maximum per significant cluster is reported. 
a SS*age > DD*age

$\int_{-4}^{-4}$
M3L

$z=-42$

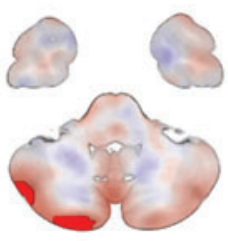

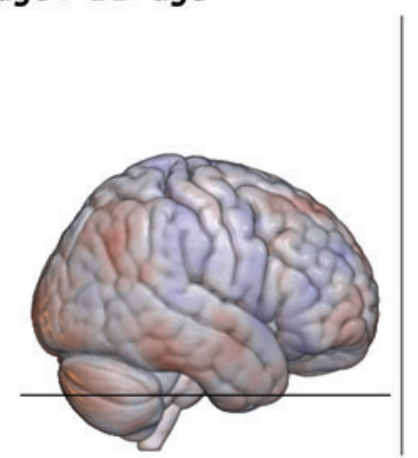

\section{b SS*age > LA*age}

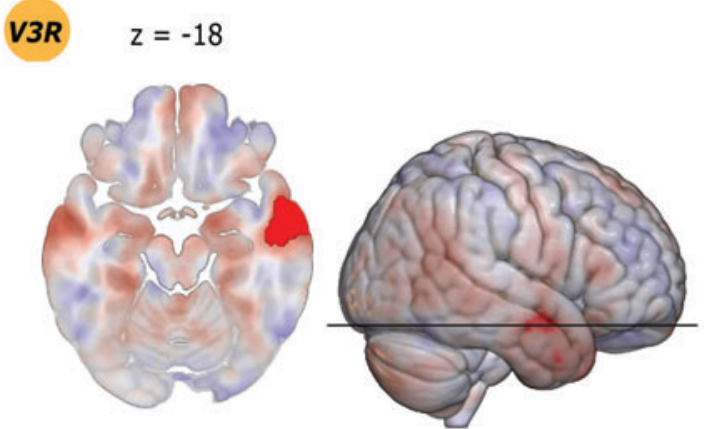

C $D D * a g e>L A * a g e$
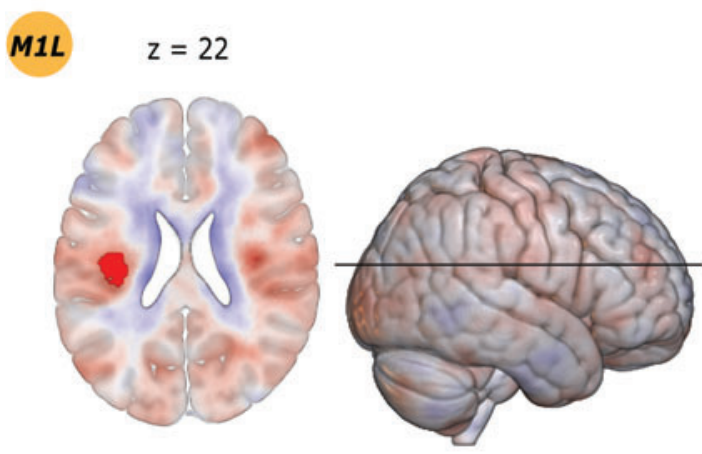

d DD*age > RA*age
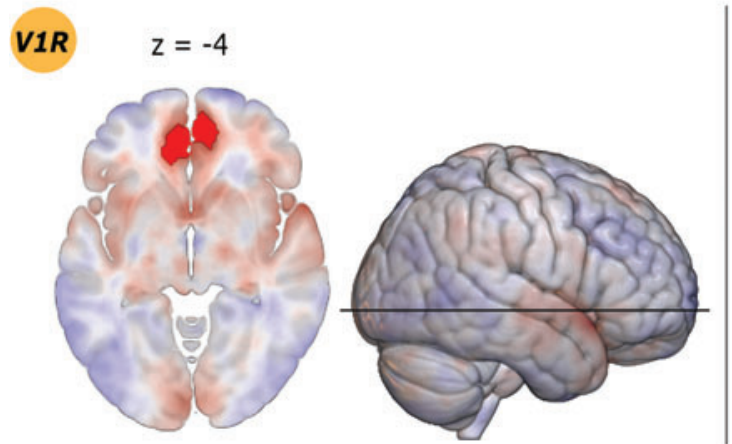

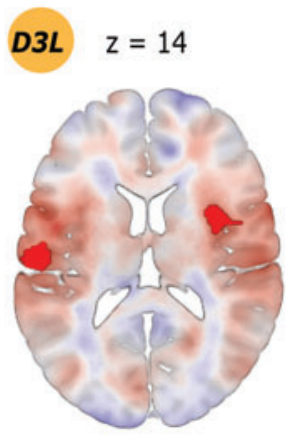

$$
\text { (23) } 2=14
$$
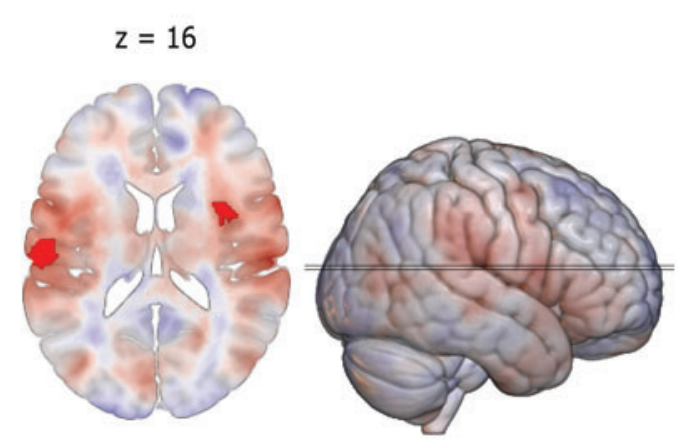

e LA*age $>R A * a g e$
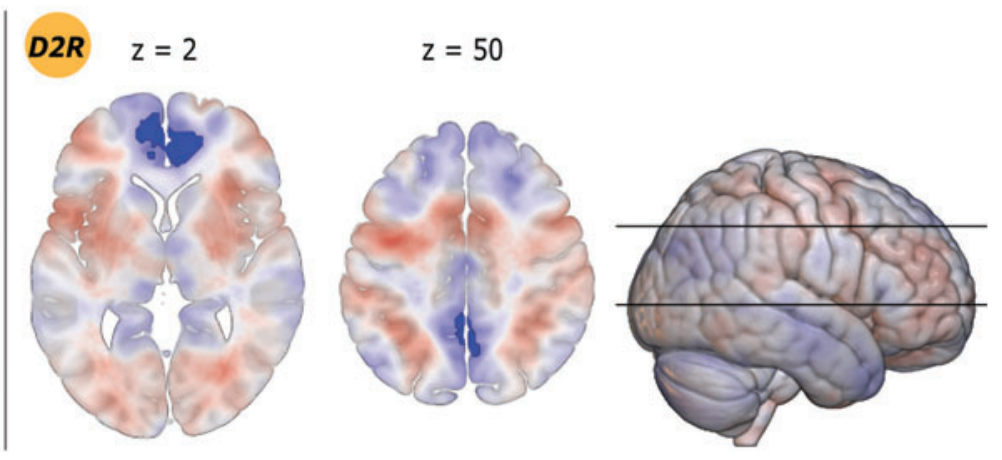

FIG. 3. Effects of age-sulcal pattern interaction on seed-based resting-state functional connectivity. Results are reported as highlighted clusters overlayed onto unthresholded T-maps; color map indicates raw $t$-values (Pernet and Madan, 2018). Significant results are shown at cluster-level FWE-corrected for multiple comparisons $p$-value $<0.00046$, and voxel-level-uncorrected $p$-value $<0.001$. Axial slices are shown with $Z$ MNI coordinates corresponding to the contrast local maxima with whole-brain height reference. Ventral seeds are labeled as V1, V2, V3; middle seeds are labeled as M1, M2, M3; dorsal seeds are labeled as D1, D2, D3. Images are in neurological display convention. Color images are available online.

\section{Double parallel/double parallel*age}

The double parallel/double parallel*age $>$ single/single* age contrast showed decreased long-distance, intrahemispheric rsFC between a left middle seed (M3 L) and the cerebellum. The double parallel/double parallel*age>leftward asymmetry*age contrast revealed increased $\mathrm{rsFC}$ of middle (M1 L) and dorsal (D3 L) seeds with the bilateral central opercular cortex. The double parallel/double parallel*age $>$ rightward asymmetry*age contrast showed increased $\mathrm{rsFC}$ between a right ventral seed (V1 R) and the ipsilateral PCG.

\section{Leftward asymmetry*age}

The leftward asymmetry*age $>$ single/single*age contrast showed decreased rsFC with increasing age between a right ventral seed (V3 R) and the temporal cortex. The leftward asymmetry*age $>$ double parallel/double parallel*age contrast revealed decreased rsFC of middle (M1 L) and dorsal (D3 L) seeds with the bilateral central opercular cortex. The leftward asymmetry*age>rightward asymmetry*age contrast showed decreased rsFC between a dorsal seed (D2 $\mathrm{R})$ and the ACC and precuneus cortex. 


\section{Rightward asymmetry ${ }^{\star}$ age}

The rightward asymmetry*age $>$ single/single*age contrast showed no significant result. The rightward asymmetry* age $>$ double parallel/double parallel*age contrast showed decreased rsFC between a right ventral seed (V1 R) and the ipsilateral PCG. The rightward asymmetry*age>leftward asymmetry*age contrast showed increased rsFC between a dorsal (D2 R) seed and the ACC and precuneus cortex.

Additional analyses with our sample split into younger (age $<35)$ and older adults (age $>60)$ are available in the Supplementary Data.

\section{Correlations between $d A C C$ connectivity and cognitive performance}

When testing for intergroup differences in correlations between connectivity values extracted from significant clusters and the scores from cognitive tests, no significant result emerged.

\section{Discussion}

We used a multimodal neuroimaging approach to investigate the influence of sulcal pattern variability on the functional organization of the dACC in a sample of healthy adults aged $20-80$ years. Our results indicate that the effects of individual differences in local morphology can alter the functional architecture of the dACC at rest, with distinct sulcal patterns associated with specific profiles of functional connectivity strength and seed-based trajectories.

When compared with the "single/single" pattern (i.e., PCS absent in both hemispheres), patterns with a PCS in the right hemisphere (i.e., "rightward asymmetry" and "double parallel/double parallel" patterns) were associated with enhanced rsFC between ventral aspects of the dACC and the rest of the brain. The "double-parallel/doubleparallel" pattern (i.e., PCS present in both hemispheres) was also associated with greater bilateral connectivity between ventral and dorsal seeds with the right angular gyrus and bilateral insulo-opercular cortices when compared with the "leftward asymmetry" pattern (i.e., PCS in the left hemisphere only), thus resulting the most connected pattern. On the contrary, the opposite "single/single" pattern was consistently linked with reduced $\mathrm{rsFC}$, with the exception of a greater connectivity with the cerebellum relative to the "double parallel/double parallel" pattern. No differences were found between patterns with and without a PCS on the right hemisphere (i.e., "rightward asymmetry" and "double parallel/double parallel" vs. "leftward asymmetry" and "single/single"). Asymmetric patterns (i.e., "leftward asymmetry" and "rightward asymmetry") were not different in terms of connectivity from each other. These results suggest profound differences between symmetric patterns (i.e., "double parallel/double parallel" vs. "single/single") and a greater incidence of enhanced connectivity for the sulcal patterns with a PCS in the right hemisphere (i.e., "double parallel/double parallel" and "rightward asymmetry").

In terms of seed-based trajectories, most target regions that showed significant differences associated with distinct sulcal patterns are key components of the salience network (SN) and default-mode network (DMN) (e.g., insular, opercular, and temporo-occipital cortices). This result is con- sistent with the central role played by the dACC as a connectivity hub in these intrinsic networks. The $\mathrm{SN}$, a paralimbic-limbic network anchored in the dACC and the anterior insula with prominent subcortical nodes, plays a central role in social behavior and contributes to the processing of relevant stimuli by integrating sensory, emotional, and cognitive information (Menon, 2015; Seeley et al., 2007). The DMN is typically activated during passive rest states or internally oriented mental processes (e.g., mind wandering, self-referential processing) (Raichle, 2015). Differences in connectivity trajectories between closely placed seeds arguably reflect fine-graded changes in the cortical cytology of the ACC (Palomero-Gallagher et al., 2008, 2019). Finally, a cross-seed investigation between SS and DD patterns revealed that intergroup differences seem to reflect more a change in terms of connectivity strength than a change in functional topology (Amiez et al., 2013).

The neurobiological mechanisms underlying the association between cortical folding morphology and rsFC are not straightforward. The regional fissurization of the cortical surface has been related to variations in intra- and inter-regional structural connectivity (Hilgetag and Barbas, 2005; Toro and Burnod, 2005; Van Essen, 1997). Therefore, it would be tempting to speculate that differences in functional connectivity profiles associated with sulcal patterns are mediated by variations in the structural connectome. However, although brain regions that are well interconnected tend to display strong patterns of functional connectivity (Segall et al., 2012; Van Den Heuvel et al., 2009), the notion of a one-to-one relationship between structural and functional brain architecture has been challenged (Lim et al., 2019; Messé et al., 2014). We thus restrict ourselves to reporting that the functional connectivity of the dACC may be associated with individual differences in local cortical morphology, suggesting a relationship that partly arises from the fetal stages of brain development when sulcal patterns are determined.

When we tested for changes of the sulcal pattern-rsFC relationship dependent on aging, we found that the effects of sulcal pattern on rsFC changed from young to older adulthood, although to a varying, pattern-specific degree. For instance, when compared with all other sulcal patterns, the "leftward asymmetry" pattern was associated with extensively diminished seed-based rsFC with increasing age. By contrast, the functional organization of individuals with a symmetric sulcal pattern (i.e., double parallel/double parallel and single/single) was remarkably conserved when compared with the other patterns. The rs-fMRI literature reports substantive changes in rsFC over the adult life span, with decreased connectivity in association networks (e.g., DMN) and increased connectivity between functionally segregated networks (e.g., DMN/frontoparietal control network/somatosensory network) (Betzel et al., 2014; Geerligs et al., 2015; Grady et al., 2016). These patterns of effects have been associated with a progressive decrease in specialization of functional systems with potential detriment to information processing in older age groups. Age-related alterations in rsFC have been associated with a number of factors, including amyloid- $\beta$ deposition (Ferreira and Busatto, 2013), neurovascular dysregulation (Galiano et al., 2020), and integrity across gray and white matter (Vidal-Piñeiro et al., 2014). Our findings suggest that sulcal pattern variability interacts with 
age-associated effects in shaping the brain's interregional connectivity shedding light on the long-term contribution of longitudinally stable neurodevelopmental markers on resting-state networks. Future longitudinal studies could investigate whether the double presence or double absence of a PCS may help individuals reach stable dACC connectivity patterns at an earlier age.

Increasing evidence shows that individual differences in dACC sulcal pattern relate to cognitive efficiency across several higher order functions that draw on cognitive control (Cachia et al., 2014; Del Maschio et al., 2019; Fornito et al., 2004). Compared with individuals with symmetric sulcal patterns, both children and adults with a leftward asymmetry pattern tend to exhibit a performance advantage on inhibitory control and working memory tasks (Borst et al., 2014; Cachia et al., 2017; Fornito et al., 2004; Huster et al., 2009; Tissier et al., 2018). The modulatory effect of sulcal pattern variability on cognitive control performance has been proposed to be mediated by differences in structural interhemispheric connectivity (Van Essen, 1997; see also Tissier et al., 2018) or functional brain activity, as hinted by task-based fMRI studies that show a topological modulation of functional responses associated with sulcation differences (Amiez et al., 2013; Crosson et al., 1999). We did not find evidence that changes in brain connectivity related to sulcal variability are also associated with differences in cognitive functions. However, since evidence for an association between sulcal variability and executive differences has been provided by other studies using different tests, a differentially modulated cognitive assessment may be required to obtain significant results.

The neurobiological interpretability of the data here presented may suffer from the lack of a univocal theory about the mechanisms regulating cortical expansion and folding (Fernández et al., 2016), representing a potential limitation to this study. The lack of a sufficient understanding of such mechanisms jeopardizes the possibility to provide finegrained mechanistic explanations for the complex interplay between early neuroanatomical constraints, functional brain activity, and cognitive performance. What the present work highlights, however, is that variability in the dACC sulcal pattern - a longitudinally stable neuroanatomical fingerprint-is associated with the functional interdependence of the $\mathrm{dACC}$ with the rest of the brain, and interacts with age-associated effects in shaping connectivity changes over adulthood. Additional research utilizing multimodal imaging and behavioral data is needed to confirm these results and further investigate the cognitive effects of structurefunction dynamics.

\section{Conclusion}

Taken together, our findings indicate that: (i) rsFC is associated with sulcal pattern variability, with distinct sulcal patterns associated with specific profiles of connectivity strength and trajectories (e.g., greater dACC connectivity associated with the presence of a PCS in the right hemisphere); (ii) the relationship between sulcal pattern and rsFC changes with increasing age, although to a varying, pattern-specific degree (e.g., more stable connectivity with increasing age for symmetric patterns and diminished connectivity with increasing age for the leftward asymmetry pattern); and (iii) cognitive effects associated with differences in structurefunction relationships have not been detected on the performed test batteries.

\section{Author Disclosure Statement}

No competing financial interests exist.

\section{Funding Information}

No funding was received for this article.

\section{Supplementary Material}

Supplementary Data

Supplementary Figure S1

Supplementary Table S1

Supplementary Table S2

\section{References}

Amiez C, Neveu R, Warrot D, Petrides M, Knoblauch K, Procyk E. 2013. The location of feedback-related activity in the midcingulate cortex is predicted by local morphology. J Neurosci 33:2217-2228.

Ashburner J, Friston KJ. 2005. Unified segmentation. Neuroimage 26:839-851.

Babayan A, Erbey M, Kumral D, et al. 2018. A mind-brain-body dataset of MRI, EEG, cognition, emotion, and peripheral physiology in young and old adults. Sci Data 6:180308.

Behzadi Y, Restom K, Liau J, Liu TT. 2007. A component based noise correction method (CompCor) for BOLD and perfusion based fMRI. Neuroimage 37:90-101.

Betzel RF, Byrge L, He Y, Goñi J, Zuo XN, Sporns O. 2014. Changes in structural and functional connectivity among resting-state networks across the human lifespan. Neuroimage 102:345-357.

Borrell V. 2018. How cells fold the cerebral cortex. J Neurosci 38:776-783.

Borst G, Cachia A, Vidal J, Simon G, Fischer C, Pineau A, et al. 2014. Folding of the anterior cingulate cortex partially explains inhibitory control during childhood: a longitudinal study. Dev Cogn Neurosci 9:126-135.

Botvinick MM, Braver TS, Barch DM, Carter CS, Cohen JD. 2001. Conflict monitoring and cognitive control. Psychol Rev 108:624-652.

Bush G, Vogt BA, Holmes J, Dale AM, Greve D, Jenike MA, Rosen BR. 2002. Dorsal anterior cingulate cortex: a role in reward-based decision making. Proc Natl Acad Sci U S A 99:523-528.

Cachia A, Borst G, Tissier C, Fisher C, Plaze M, Gay O, et al. 2016. Longitudinal stability of the folding pattern of the anterior cingulate cortex during development. Dev Cogn Neurosci 19:122-127.

Cachia A, Borst G, Vidal J, Fischer C, Pineau A, Mangin JF, Houdé O. 2014. The shape of the ACC contributes to cognitive control efficiency in preschoolers. J Cogn Neurosci 26:96-106.

Cachia A, Del Maschio N, Borst G, Della Rosa PA, Pallier C, Costa A, et al. 2017. Anterior cingulate cortex sulcation and its differential effects on conflict monitoring in bilinguals and monolinguals. Brain Lang 175:57-63.

Chai XJ, Castañón AN, Öngür D, Whitfield-Gabrieli S. 2012. Anticorrelations in resting state networks without global signal regression. Neuroimage 59:1420-1428.

Chen PY, Chiou JM, Yang YF, Chen YT, Hsieh HL, Chang YL, Tseng WY. I. 2016. Heterogeneous aging effects on 
functional connectivity in different cortical regions: a resting-state functional mri study using functional data analysis. PLoS One 11:e0162028.

Crosson B, Sadek JR, Bobholz JA, Gökçay D, Mohr CM, Leonard CM, et al. 1999. Activity in the paracingulate and cingulate sulci during word generation: an fMRI study of functional anatomy. Cereb Cortex 9:307-316.

Damoiseaux JS. 2017. Effects of aging on functional and structural brain connectivity. Neuroimage 160:32-40.

Del Maschio N, Sulpizio S, Fedeli D, Ramanujan K, Ding G, Weekes BS, et al. 2019. ACC sulcal patterns and their modulation on cognitive control efficiency across lifespan: a neuroanatomical study on bilinguals and monolinguals. Cereb Cortex 29:3091-3101.

Fernández V, Llinares-Benadero C, Borrell V. 2016. Cerebral cortex expansion and folding: what have we learned? EMBO J 35:1021-1044.

Ferreira LK, Busatto GF. 2013. Resting-state functional connectivity in normal brain aging. Neurosci Biobehav Rev 37:384-400.

Fornito A, Yücel M, Wood S, Stuart GW, Buchanan JA, Proffitt T, et al. 2004. Individual differences in anterior cingulate/ paracingulate morphology are related to executive functions in healthy males. Cereb cortex 14:424-431.

Galiano A, Mengual E, de Eulate RG, Galdeano I, Vidorreta M, Recio M, et al. 2020. Coupling of cerebral blood flow and functional connectivity is decreased in healthy aging. Brain Imaging Behav 14:436-450.

Garrison JR. 2017. Paracingulate Sulcus Measurement Protocol. https://www.repository.cam.ac.uk/bitstream/handle/1810/ 264520/PCS\%20Measurement $\% 20$ Protocol.pdf?sequence= 1\&isAllowed=y Last accessed June 10, 2019.

Garrison JR, Fernyhough C, McCarthy-Jones S, Haggard M, Simons JS. 2015. Paracingulate sulcus morphology is associated with hallucinations in the human brain. Nat Commun 6: 8956.

Geerligs L, Renken RJ, Saliasi E, Maurits NM, Lorist MM. 2015. A brain-wide study of age-related changes in functional connectivity. Cereb Cortex 25:1987-1999.

Grady C, Sarraf S, Saverino C, Campbell K. 2016. Age differences in the functional interactions among the default, frontoparietal control, and dorsal attention networks. Neurobiol Aging 41:159-172.

Hilgetag CC, Barbas H. 2005. Developmental mechanics of the primate cerebral cortex. Anat Embryol (Berl) 210:411.

Huster RJ, Westerhausen R, Kreuder F, Schweiger E, Wittling W. 2007. Morphologic asymmetry of the human anterior cingulate cortex. Neuroimage 34:888-895.

Huster RJ, Wolters C, Wollbrink A, Schweiger E, Wittling W, Pantev C, Junghofer M. 2009. Effects of anterior cingulate fissurization on cognitive control during stroop interference. Hum Brain Mapp 30:1279-1289.

Jahn A, Nee DE, Alexander WH, Brown JW. 2016. Distinct regions within medial prefrontal cortex process pain and cognition. J Neurosci 36:12385-12392.

Kochunov P, Mangin JF, Coyle T, Lancaster J, Thompson P, Rivière D, et al. 2005. Age-related morphology trends of cortical sulci. Hum Brain Mapp 26:210-220.

Leonard CM, Towler S, Welcome S, Chiarello C. 2009. Paracingulate asymmetry in anterior and midcingulate cortex: sex differences and the effect of measurement technique. Brain Struct Funct 213:553-569.

Li S, Han Y, Wang D, Yang H, Fan Y, Lv Y, et al. 2010. Mapping surface variability of the central sulcus in musicians. Cereb Cortex 20:25-33.
Li S, Xia M, Pu F, Li D, Fan Y, Niu H, et al. 2011. Age-related changes in the surface morphology of the central sulcus. Neuroimage 58:381-390.

Lim S, Radicchi F, van den Heuvel MP, Sporns O. 2019. Discordant attributes of structural and functional brain connectivity in a two-layer multiplex network. Sci Rep 9: 2885.

Lohmann G, Von Cramon DY, Steinmetz H. 1999. Sulcal variability of twins. Cereb Cortex 9:754-763.

Lopez-Persem A, Verhagen L, Amiez C, Petrides M, Sallet J. 2019. The human ventromedial prefrontal cortex: sulcal morphology and its influence on functional organization. J Neurosci 39:3627-3639.

Margulies DS, Kelly AC, Uddin LQ, Biswal BB, Castellanos FX, Milham MP. 2007. Mapping the functional connectivity of anterior cingulate cortex. Neuroimage 37:579-588.

Menon V. 2015. Salience network. In: Toga AW (ed.) Brain Mapping: An Encyclopedic Reference, vol. 2. London: Academic Press: Elsevier; pp. 597-611.

Messé A, Rudrauf D, Benali H, Marrelec G. 2014. Relating structure and function in the human brain: relative contributions of anatomy, stationary dynamics, and non-stationarities. PLoS Comput Biol 10:e1003530.

Mikhael S, Hoogendoorn C, Valdes-Hernandez M, Pernet C. 2018. A critical analysis of neuroanatomical software protocols reveals clinically relevant differences in parcellation schemes. Neuroimage 170:348-364.

Nieto-Castañón A. 2020. Handbook of Functional Connectivity Magnetic Resonance Imaging Methods in CONN. Boston, MA: Hilbert Press.

Ono M, Kubik S, Abernathy CD. 1990. Atlas of Cerebral Sulci. New York: Gečrg Thieme Verlag Stuttgart.

Palomero-Gallagher N, Hoffstaedter F, Mohlberg H, Eickhoff SB, Amunts K, Zilles K. 2019. Human pregenual anterior cingulate cortex: structural, functional, and connectional heterogeneity. Cereb Cortex 29:2552-2574.

Palomero-Gallagher N, Mohlberg H, Zilles K, Vogt B. 2008. Cytology and receptor architecture of human anterior cingulate cortex. J Comp Neurol 508:906-926.

Paus T, Tomaiuolo F, Otaky N, MacDonald D, Petrides M, Atlas J, et al. 1996. Human cingulate and paracingulate sulci: pattern, variability, asymmetry, and probabilistic map. Cereb Cortex 6:207-214.

Pernet CR, Madan CR. 2020, Data visualization for inference in tomographic brain imaging. Eur J Neurosci 51:695705.

Pernet CR, Wilcox RR, Rousselet GA. 2013. Robust correlation analyses: false positive and power validation using a new open source matlab toolbox. Front Psychol 3: 606.

Power JD, Barnes KA, Snyder AZ, Schlaggar BL, Petersen SE. 2012. Spurious but systematic correlations in functional connectivity MRI networks arise from subject motion. Neuroimage 59:2142-2154.

Raichle ME. 2015. The restless brain: how intrinsic activity organizes brain function. Philos Trans R Soc Lond B Biol Sci 370:20140172.

Rousselet GA, Pernet CR. 2012. Improving standards in brain-behavior correlation analyses. Front Hum Neurosci 6:119.

Seeley WW, Menon V, Schatzberg AF, Keller J, Glover GH, Kenna H, et al. 2007. Dissociable intrinsic connectivity networks for salience processing and executive control. J Neurosci 27:2349-2356. 
Segall JM, Allen EA, Jung RE, Erhardt EB, Arja SK, Kiehl KA, Calhoun VD. 2012. Correspondence between structure and function in the human brain at rest. Front Neuroinform 6:10.

Smith SM, Jenkinson M, Woolrich MW, Beckmann CF, Behrens TE, Johansen-Berg H, et al. 2004. Advances in functional and structural MR image analysis and implementation as FSL. Neuroimage 23:S208-S219.

Sheth SA, Mian MK, Patel SR, Asaad WF, Williams ZM, Dougherty DD, et al. 2012. Human dorsal anterior cingulate cortex neurons mediate ongoing behavioural adaptation. Nature 488:218-221.

Tissier C, Linzarini A, Allaire-Duquette G, Mevel K, Poirel N, Dollfus S, et al. 2018. Sulcal polymorphisms of the IFC and ACC contribute to inhibitory control variability in children and adults. eNeuro 5:ENEURO.0197-17.2018.

Toro R, Burnod Y. 2005. A morphogenetic model for the development of cortical convolutions. Cereb Cortex 15:19001913.

Van Den Heuvel MP, Stam CJ, Kahn RS, Pol HEH. 2009. Efficiency of functional brain networks and intellectual performance. J Neurosci 29:7619-7624.

Van Essen DC. 1997. A tension-based theory of morphogenesis and compact wiring in the central nervous system. Nature 385:313-318.

Vidal-Piñeiro D, Valls-Pedret C, Fernández-Cabello S, ArenazaUrquijo EM, Sala-Llonch R, Solana E, et al. 2014. Decreased default mode network connectivity correlates with ageassociated structural and cognitive changes. Front Aging Neurosci 6:256.
Vogt BA, Palomero-Gallagher N. 2012. Cingulate cortex. In: Mai JK, Paxinos G (eds.) The Human Nervous System. Cambridge, MA: Academic Press; pp. 947-987.

Wei X, Yin Y, Rong M, Zhang J, Wang L, Wu Y, et al. 2017. Paracingulate sulcus asymmetry in the human brain: effects of sex, handedness, and race. Sci Rep 7:42033.

Whitfield-Gabrieli S, Nieto-Castanon A. 2012. Conn: a functional connectivity toolbox for correlated and anticorrelated brain networks. Brain Connect 2:125-141.

Wilcox R. 2004. Inferences based on a skipped correlation coefficient. J Appl Stat 31:131-143.

Wilcox RR. 1994. The percentage bend correlation coefficient. Psychometrika 59:601-616.

Yücel M, Stuart GW, Maruff P, Velakoulis D, Crowe SF, Savage G, Pantelis C. 2001. Hemispheric and gender-related differences in the gross morphology of the anterior cingulate/ paracingulate cortex in normal volunteers: an MRI morphometric study. Cereb Cortex 11:17-25.

Address correspondence to: Jubin Abutalebi

Centre for Neurolinguistics and Psycholinguistics (CNPL) Università Vita-Salute San Raffaele Via Olgettina, 58 Milano 20132 Italy

E-mail: abutalebi.jubin@hsr.it 